\title{
On Invariants of Hirzebruch and Cheeger-Gromov
}

\author{
Stanley Chang \\ ShMuel WeInBerger \\ Department of Mathematics, Wellesley College \\ Wellesley, MA 02481, USA \\ and \\ Department of Mathematics, University of Chicago \\ Chicago, IL 60637, USA
}

Email: shmuel@math.uchicago.edu, sschang@palmer.wellesley.edu

\begin{abstract}
We prove that, if $M$ is a compact oriented manifold of dimension $4 k+3$, where $k>0$, such that $\pi_{1}(M)$ is not torsion-free, then there are infinitely many manifolds that are homotopic equivalent to $M$ but not homeomorphic to it. To show the infinite size of the structure set of $M$, we construct a secondary invariant $\tau_{(2)}: S(M) \rightarrow \mathbb{R}$ that coincides with the $\rho$-invariant of Cheeger-Gromov. In particular, our result shows that the $\rho$-invariant is not a homotopy invariant for the manifolds in question.
\end{abstract}

AMS Classification numbers Primary: 57R67

Secondary: 46L $80,58 \mathrm{G} 10$

Keywords: Signature, $L^{2}$-signature, structure set, $\rho$-invariant

Proposed: Stephen Ferry

Seconded: Ronald Stern, Benson Farb

(C) Geometry $\& \mathcal{G}$ Topology $\mathcal{P}$ ublications
Received: 28 March 2003

Accepted: 30 April 2003 
In their analysis of free involutions on the sphere, Browder and Livesay [4] introduced an invariant which they used to study the existence and uniqueness question for desuspensions. In particular, they produced infinitely many manifolds homotopy equivalent, but not diffeomorphic or even homeomorphic, to $\mathbb{R P}^{4 k-1}$ for $k>1$. Hirzebruch [11] soon gave an alternative definition of this invariant as follows: Any manifold $M$ of dimension $4 k-1$ with $\pi_{1}(M)=\mathbb{Z}_{2}$ has a multiple $\ell M$ which bounds some $W^{4 k}$ such that $\pi_{1}(M) \rightarrow \pi_{1}(W)$ is an isomorphism. Define

$$
\tau(M)=\frac{1}{\ell} \cdot g-\operatorname{sig}(\widetilde{W})=\frac{1}{\ell}\left(\left.\operatorname{tr} g\right|_{H_{+}}-\left.\operatorname{tr} g\right|_{H_{-}}\right)=\frac{1}{\ell}(\operatorname{sig}(\widetilde{W})-2 \operatorname{sig}(W)),
$$

where $g \in \mathbb{Z}_{2}$ is the nontrivial element and $\widetilde{W}$ is the two-fold cover of $W$ with $H_{ \pm} \equiv H_{ \pm}^{2 k}(\widetilde{W})$. The fact that $\tau(M)$ is independent of $W$ can be seen either using the $G$-signature formula [2] or by a bordism argument.

Remark Actually, Hirzebruch's invariant was only shown a bit later to be equal (up to a sign) to the Browder-Livesay invariant by Hirzebruch and Lopez de Medrano [13].

It is quite simple to modify $\tau$ to obtain invariants of arbitrary $M^{4 k-1}$ with finite fundamental group $G=\pi_{1}(M)$ by using the $G$-signature of $\widetilde{W}$ modulo the regular representation (as in [22]) or by using characters equal to $\left.\operatorname{tr} g\right|_{H_{+}-}$ $\left.\operatorname{tr} g\right|_{H_{-}}$for any $g \neq e$. As a very special and important case, one can even simply use $\operatorname{sig}(\widetilde{W})-|G| \operatorname{sig}(W)$ which equals $\sum_{g \neq e} \chi_{g}\left(\operatorname{sig}_{G}(W)\right)$ to obtain a quite useful invariant.

As an application of their index theorem for manifolds with boundary, Atiyah, Patodi and Singer [2] obtain such invariants of $M$ without introducing $W$. More precisely, the APS invariant requires the use of an auxiliary Riemannian metric on $M$, but their index theorem for manifolds with boundary shows that the number obtained is independent of this metric. All of the numerical invariants mentioned above are differences of $\eta$-invariants (which measure "spectral asymmetry") for the signature operator twisted by suitable flat bundles.

The APS method admits extensive generalization to the case in which $\pi_{1}(M)$ is possibly infinite. In [1] Atiyah generalized the index theorem from compact closed manifolds to universal covers of compact manifolds (or even manifolds with proper actions of a discrete group). Using von Neumann traces, he defined

$$
\operatorname{ind}_{\Gamma}(\widetilde{D})=\operatorname{dim}_{\Gamma}\left(\operatorname{ker} \widetilde{\not D} \text { on } L^{2}(\widetilde{M})\right)-\operatorname{dim}_{\Gamma}\left(\operatorname{coker} \widetilde{D} \text { on } L^{2}(\widetilde{M})\right) \text {. }
$$

Both [14] and [15] are excellent references for ideas related to the von Neumann trace and its applications in geometry and topology. 
Note Atiyah showed that $\operatorname{ind}_{\Gamma}(\widetilde{D})=$ ind $(\not D)$, which generalizes the multiplicativity property of ordinary indices for finite coverings (see appendix). This result is quite remarkable since, if the group is infinite, the elements of ker $\not D$ never lift to be $L^{2}$ on the universal cover, but they nonetheless lead indirectly to elements of the $L^{2}$ kernel (at the level of indices, of course). Cheeger and Gromov $[5,6]$ introduced the analogue of the APS invariant in this setting: namely they studied an $L^{2}$-invariant $\eta_{(2)}(\widetilde{M})$ of compact $M$ and the difference $\rho_{(2)}(M)=\eta_{(2)}(\widetilde{M})-\eta(M)$ which is metric independent. For $\Gamma=\mathbb{Z}_{2}$ the quantity $\rho_{(2)}(M)$ is simply the Hirzebruch invariant or the Browder-Livesay invariant.

Thom's classical work on cobordism implies that every compact odd dimensional oriented manifold $M$ has a multiple $r M$ which is the boundary of an oriented manifold $W$. Hausmann [9] showed furthermore that, for every such $M$ with fundamental group $\Gamma$, there is a manifold $W$ such that $\Gamma$ injects into $\pi_{1}(W)$ and $\partial W=r M$, for some multiple $r M$ of $M$; that is, he showed that nullcobordant manifolds bound in such a way that their fundamental groups inject. If $\Gamma$ is a group, we can define the $L^{2}$-signature $\operatorname{sig}_{(2)}^{\Gamma}(V)=\operatorname{dim}_{\Gamma}\left(V^{+}\right)-\operatorname{dim}_{\Gamma}\left(V^{-}\right)$for any $\ell^{2}(\Gamma)$-module $V$ endowed with nonsingular symmetric bilinear form. As usual, one uses an inner product to rewrite the symmetric bilinear form in terms of a self-adjoint operator, and then uses the spectral theorem to obtain projections to the positive and negative definite part. If $N^{4 k}$ is a $\Gamma$-space, let $\operatorname{sig}_{(2)}^{\Gamma}(N)$ be given by the $L^{2}$-signature of the symmetric form induced by cap product on its middle cohomology.

Define a new "Hirzebruch type" invariant $\tau_{(2)}$ given by

$$
\tau_{(2)}^{G}(M)=\frac{1}{r}\left(\operatorname{sig}_{(2)}^{G}\left(W_{G}\right)-\operatorname{sig}(W)\right),
$$

where $G=\pi_{1}(W)$ and $W_{G}$ is the induced $G$-cover of $W$. Suppose that $r M=\partial W$ with $\pi_{1}(M)=\Gamma$ injecting into $\pi_{1}(W)=G$. If $G$ injects into some larger group $G^{\prime}$, and let $W_{G^{\prime}}$ be the $G^{\prime}$-space induced from the $G$ action on $W_{G}$ to $G^{\prime}$. Notice that $\partial\left(W_{G^{\prime}} / G^{\prime}\right)=r M$, so we can use the larger group $G^{\prime}$ to define $\tau_{(2)}$. However, by the $\Gamma$-induction property of Cheeger-Gromov $[6$, page 8 , equation (2.3)], we have

$$
\begin{aligned}
\tau_{(2)}^{G^{\prime}}(M) & =\frac{1}{r}\left(\operatorname{sig}_{(2)}^{G^{\prime}}\left(W_{G^{\prime}}\right)-\operatorname{sig}\left(W_{G^{\prime}} / G^{\prime}\right)\right) \\
& =\frac{1}{r}\left(\operatorname{sig}_{(2)}^{G}(W)-\operatorname{sig}(W)\right) \\
& =\tau_{(2)}^{G}(M) .
\end{aligned}
$$

So one can pass to any larger group without changing the value of the invariant. Now, given two manifolds $W$ and $W^{\prime}$ with the required bounding properties, 
we can use the large group $G^{\prime}=\pi_{1}(W) *_{\Gamma} \pi_{1}\left(W^{\prime}\right)$, which contains both fundamental groups, and the usual Novikov additivity argument, to see that $\tau_{(2)}$ is independent of all choices.

In fact, according to [16], we have $\tau_{(2)}=\rho_{(2)}$, but we do not need this equality for the proofs of our main theorems. The goal of this paper is to prove a few theorems about $\tau_{(2)}(M)$ (or $\rho_{(2)}(M)$ ) that extend or are analogues of the classically understood situation for finite fundamental group.

Theorem 1 For any compact oriented manifold $M^{4 k-1}$, where $k \geq 2$, such that $\pi_{1}(M)$ is not torsion-free, there exist infinitely many manifolds $M_{i}$ which are simple homotopy equivalent and tangentially equivalent to $M$, but not homeomorphic to $M$. If $M$ is smooth, the $M_{i}$ can be taken to be smooth as well. This infinite number is detected by $\tau_{(2)}$.

\section{Remarks}

(1) Very similar arguments to the proof of Theorem 1 show that any $M^{4 k+2}$ with non-torsion-free fundamental group has infinite diffeotopy group.

(2) The analogue for $M^{4 k+1}$ is false. For instance, the real projective space $\mathbb{R} \mathbb{P}^{4 k+1}$ has a finite structure set. See [13]. Also, we cannot dispense with the orientability hypothesis as $\mathbb{R} \mathbb{P}^{2} \times S^{5}$ has finite structure set.

(3) There are predictions in the literature about $L_{4 k}(\Gamma) \otimes \mathbb{Q}$ and its expression in terms of the group homology of normalizers of finite subgroups. See [8] and page 261 of [24]. These would suggest that Theorem 1 is true except for the thorny problem of self-homotopy equivalences of $M$. These maps $h: M \rightarrow M$ could represent nontrivial elements of the structure set $S(M)$, but nonetheless they are not the elements described in Theorem 1.

(4) If $M$ is smooth, all the $M_{i}$ can be taken smooth as well, without even being homeomorphic. The extension of $\tau_{(2)}$ from smooth to topological manifolds is not very hard by bordism methods. After multiplication by a positive integer, every topological manifold is cobordant to a smooth manifold $N$ via a cobordism $V$ (all with the same fundamental group), so we can consider $\tau_{(2)}(N)-(\operatorname{sig}(2)-\operatorname{sig})(V)$.

(5) The theorem above asserts that, whenever $\pi_{1}(M)$ has torsion, then $\tau_{(2)}$ is not a homotopy invariant. Mathai [18] conjectured that the converse is true for $\rho_{(2)}$ and proved it for torsion-free crystallographic fundamental group. Thus, the above result is the converse to Mathai's conjecture. 
Mathai's conjecture is an analogue of a conjecture and theorem of the second author [23] for Atiyah-Patodi-Singer invariants. Keswani [12] proved a general homotopy invariance theorem: if the assembly map $K_{*}(B \Gamma) \rightarrow K_{*}\left(C_{\max }^{*} \Gamma\right)$ is surjective, then $\rho_{(2)}$ is a homotopy invariant for manifolds with fundamental group $\Gamma$. This hypothesis holds for fundamental group of real and complex hyperbolic manifolds for torsion-free amenable groups by the theorem of Higson and Kasparov [10]. However, it fails for all nontrivial groups satisfying property $T$, ie, for many torsion-free lattices in higher rank or in $\operatorname{Sp}(n, 1)$. Our next theorem somewhat repairs this problem.

Theorem 2 If $\Gamma$ is a torsion-free discrete subgroup of $S L_{n}(\mathbb{R})$, then $\rho$ is a homotopy invariant for manifolds with fundamental group $\Gamma$. More generally, if $\Gamma=\pi_{1}(M)$ is residually finite and the Borel conjecture holds for $\Gamma$, then $\rho_{(2)}$ is a homotopy invariant (see eg [8]).

In a future paper, the first author plans to remove the residual finiteness hypotheses arising in this work.

We now turn to the proof of Theorem 1. Let $M$ be as in the theorem and $\mathbb{Z}_{n} \subset \pi_{1}(M)=\Gamma$ be a nontrivial cyclic subgroup. The $M_{i}$ are just the results of acting by elements of $\operatorname{im}\left(L_{4 k}\left(\mathbb{Z}_{n}\right) \rightarrow L_{4 k}(\Gamma)\right)$ on $S(M)$ in the surgery exact sequence

$$
\cdots \rightarrow H_{4 k}(M, \mathbb{L}) \rightarrow L_{4 k}(\Gamma) \rightarrow S(M) \rightarrow H_{4 k-1}(M, \mathbb{L}) \rightarrow L_{4 k-1}(\Gamma) \rightarrow \cdots
$$

The image of $A: H_{4 k}(M, \mathbb{L}) \rightarrow L_{4 k}(\Gamma)$ consists of quadratic forms arising from closed manifolds and for such manifolds we have $\operatorname{sig}_{(2)}^{\Gamma}(N)=\operatorname{sig}(N)$ by Atiyah [1]. Note that this higher signature may be viewed as a map $\operatorname{sig}_{(2)}^{\Gamma}: L_{4 k}(\Gamma) \rightarrow \mathbb{R}$ (see [22] for the definition of $L$-groups). This image must equal $\operatorname{ker}\left(L_{4 k}(\Gamma) \rightarrow\right.$ $S(M))$, the set of all $a \in L_{4 k}(\Gamma)$ for which $a(M)=M$ diffeomorphically. ${ }^{1}$ If $a \in L_{4 k}(\Gamma)$, let $V_{a}$ be the form representing it. Let $\alpha_{\Gamma}: L_{4 k}(\Gamma) \rightarrow \mathbb{R}$ be given by the homomorphism $\alpha_{\Gamma}(V) \equiv \operatorname{sig}_{(2)}{ }_{(V)}(V)-\operatorname{sig}(V)$. Hence, if $a(M)=M$, then $\alpha_{\Gamma}\left(V_{a}\right)=0$. We would like to produce infinitely many $V_{a^{\prime}}$ such that $\alpha_{\Gamma}\left(V_{a^{\prime}}\right) \neq 0$.

To show that the homomorphism $\alpha_{\Gamma}: L_{4 k}(\Gamma) \rightarrow \mathbb{R}$ has infinite image, it suffices to show that it is nontrivial. Let $p_{*}: L_{4 k}\left(\mathbb{Z}_{n}\right) \rightarrow L_{4 k}(\Gamma)$ be the map induced

\footnotetext{
${ }^{1}$ More precisely, the equality represents a diffeomorphism homotopic to the homotopy equivalence implicit in the fact that $a(M)$ is a member of a structure set.
} 
by the injection $p: \mathbb{Z}_{n} \rightarrow \Gamma$. By the induction property of $\Gamma$-dimension, we have the commutative diagram



so we only have to check that $\alpha_{\mathbb{Z}_{n}}: L_{4 k}\left(\mathbb{Z}_{n}\right) \rightarrow \mathbb{R}$ is nontrivial. We note that, inverting 2 , it makes no difference whether the coefficient ring is $\mathbb{Z}$ or $\mathbb{Q}[20]$ or whether one works with projective, free or based-free modules (by the wellknown "Rothenberg exact sequences"). Thus, we need only produce a bilinear form on a projective module over $\mathbb{Q Z}_{n}$, namely the one-by-one bilinear form [1] on the module $\mathbb{Q}$, which is projective over $\mathbb{Q Z}_{n}$. By definition, our invariant is $\frac{1}{n}-1$ for this element, which is always nontrivial for $n>1$.

If $r M$ cobounds some space $W$ for which $\pi_{1}(M)$ injects into $G=\pi_{1}(W)$, we can extend each boundary component $M$ by the cobordism $Y$, so that $r M^{\prime}$ cobounds $W^{\prime}$, where $W^{\prime}$ is obtained from $W$ and $r$ copies of $Y$. Hence by Novikov additivity and $\Gamma$-induction, we have

$$
\begin{aligned}
\tau_{(2)}^{G}(M)-\tau_{(2)}^{G}\left(M^{\prime}\right)= & \frac{1}{r}\left(\operatorname{sig}_{(2)}^{G}\left(W_{G}\right)-\operatorname{sig}(W)\right)-\frac{1}{r}\left(\operatorname{sig}_{(2)}^{G}\left(W_{G}^{\prime}\right)-\operatorname{sig}\left(W^{\prime}\right)\right) \\
= & \frac{1}{r}\left(\operatorname{sig}_{(2)}^{G}\left(W_{G}\right)-\operatorname{sig}(W)\right)-\frac{1}{r}\left(\operatorname{sig}_{(2)}^{G}(W)\right) \\
& \quad+r \cdot \operatorname{sig}_{(2)}^{G}\left(Y_{G}-\operatorname{sig}(W)-r \cdot \operatorname{sig}(Y)\right) \\
= & \operatorname{sig}_{(2)}^{G}\left(Y_{G}\right)-\operatorname{sig}(Y) \\
= & \operatorname{sig}_{(2)}^{\Gamma}\left(Y_{\Gamma}\right)-\operatorname{sig}(Y) \\
= & \alpha_{\Gamma}(V),
\end{aligned}
$$

where $V \in L_{4 k}(\Gamma)$ represents the cobordism $Y$.

Let $V_{a} \in L_{4 k}(\Gamma)$ such that $\alpha_{\Gamma}\left(V_{a}\right) \neq 0$, where $\Gamma=\pi_{1}(M)$. Since $(M \rightarrow M) \in$ $S_{4 k-1}(M)$, there is a cobordism $Y_{a}$ such that $\partial Y_{a}=M \cup M^{\prime}$ representing $V_{a}$. Clearly $M$ and $M^{\prime}$ are homotopy equivalent but not diffeomorphic. Consider $V_{a_{i}} \in L_{4 k}(\Gamma)$ for all $i \in \mathbb{N}$ such that $\alpha_{\Gamma}\left(V_{a_{i}}\right) \neq \alpha_{\Gamma}\left(V_{a_{j}}\right)$ for all $i \neq j$ and all nonzero. Let $M_{i}$ be the result of acting on $M$ by $V_{a_{i}}$. Then $\tau_{(2)}^{G}(M)-\tau_{(2)}^{G}\left(M_{i}\right)=$ $\alpha_{\Gamma}\left(V_{a_{i}}\right) \neq 0$. Also

$$
\begin{aligned}
\tau_{(2)}^{G}\left(M_{i}\right)-\tau_{(2)}^{G}\left(M_{j}\right) & =\tau_{(2)}^{G}\left(M_{i}\right)-\tau_{(2)}^{G}(M)+\tau_{(2)}^{G}(M)-\tau_{(2)}^{G}\left(M_{j}\right) \\
& =-\alpha_{\Gamma}\left(V_{\alpha_{i}}\right)+\alpha_{\Gamma}\left(V_{\alpha_{j}}\right) \neq 0,
\end{aligned}
$$


as desired.

Now we prove Theorem 2. For a finite group $\Gamma$, the rho-invariant $\rho_{(2)}=\eta_{(2)}-\eta$ is equal to $\frac{1}{|\Gamma|} \eta_{\otimes \mathbb{R} \Gamma}-\eta$, where $\eta_{\otimes \mathbb{R} \Gamma}$ is the eta-invariant for $M$ with coefficients in the flat bundle associated to the regular representation. According to [17], if $\Gamma$ is residually finite with descending quotients $\Gamma_{k}$, then

$$
\rho_{(2)}=\lim _{k \rightarrow \infty}\left(\frac{1}{\left|\Gamma_{k}\right|}\left(\eta_{\otimes \mathbb{R} \Gamma}-\eta\right)\right) .
$$

Indeed, Cheeger and Gromov introduced $\rho_{(2)}$ to generalize the right-hand side to non-residually finite fundamental group. However, according to [24], for any representation $V$ of a group satisfying the Borel conjecture, the quantity $\frac{1}{\operatorname{dim} V} \eta_{\otimes V}-\eta$ is a homotopy invariant. Since $\rho$ is the limit of homotopy invariants, a fortiori, it too is a homotopy invariant.

If $\Gamma$ is a discrete subgroup of $\mathrm{SL}_{n}(\mathbb{R})$, then it is a classical lemma of Selberg that $\Gamma$ is residually finite. The fact that the Borel conjecture holds if $\Gamma$ is in addition torsion-free is the main result of [8].

Problem If $\pi_{1}(M)$ has many conjugacy classes of elements of finite order, then one would expect that $S(M)$ is rather larger than the $\mathbb{Z}$ we just detected. Can this statement be proven unconditionally? Wolfgang Lück has pointed out that, using the traces associated to elements of finite order that have finitely many conjugates (eg, central elements), one can extend Theorem 1 and detect a larger $S(M)$. But this result is still rather smaller than the predicted size of the structure set.

\section{Appendix: Acyclic groups and Atiyah's theorem}

For the convenience of the reader we shall prove here the theorems of Atiyah and Hausmann invoked in the paper. Let $M$ be a closed manifold. According to [3], there is an acyclic group $A$ with an injective homomorphism $h: \pi_{1}(M) \rightarrow A$. Since $A$ is acyclic, the map $\Omega_{n}(K(A, 1)) \rightarrow \Omega_{n}(*)$ is an isomorphism, so that, if $M$ bounds (ie, $M$ is zero in $\Omega_{n}(*)$ ), it bounds a manifold $W$ such that



commutes; a fortiori $\pi_{1}(M) \rightarrow \pi_{1}(W)$ is injective. This argument gives Hausmann's result. 
Now for Atiyah's theorem we observe, by the induction and bordism invariance

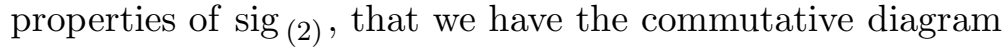

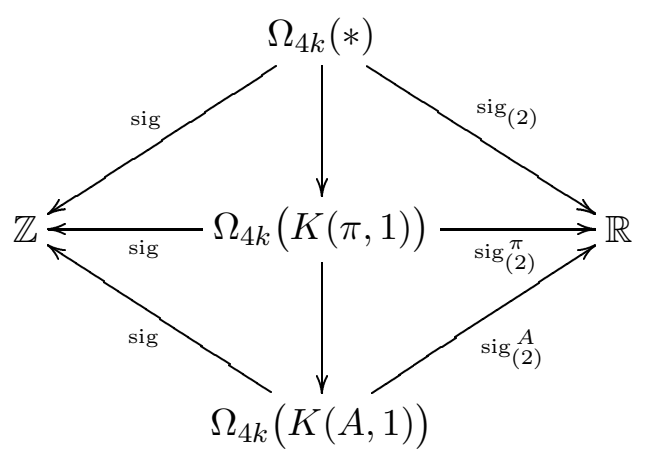

Since $\Omega_{4 k}(*) \rightarrow \Omega_{4 k}(K(A, 1))$ is an isomorphism and sig $=\operatorname{sig}(2)$ for the trivial group, the conclusion follows.

Acknowledgements We would like to thank both Wolfgang Lück and the referee for their helpful comments.

Stanley Chang was partially supported by NSF Grant DMS-9971657; Shmuel Weinberger was also partially supported by an NSF grant.

\section{References}

[1] M Atiyah, Elliptic operators, discrete groups and von Neumann algebras, Astérisque 32-33 (1976) 43-72

[2] M Atiyah, V Patodi, I Singer, Spectral asymmetry and Riemannian geometry, I. Math. Proc. Cambridge Philos. Soc. 77 (1975) 43-69, II. Math. Proc. Cambridge Philos. Soc. 78 (1975) 405-432, III. Math. Proc. Cambridge Philos. Soc. 79 (1976) 71-99

[3] G Baumslag, E Dyer, A Heller, The topology of discrete groups, J. Pure Applied Alg. 16 (1980) 1-47

[4] W Browder, R Livesay, Fixed point free involutions on homotopy spheres, Tôhoku Math. J. (2) 25 (1973) 69-87

[5] J Cheeger, M Gromov, On the characteristic numbers of complete manifolds of bounded curvature and finite volume, Differential Geometry and Complex Analysis, 115-154, Springer, Berlin-New York (1985)

[6] J Cheeger, M Gromov, Bounds on the von Neumann dimension of $L^{2}-$ cohomology and the Gauss-Bonnet theorem for open manifolds, J. Differential Geom. 21 (1985) 1-34 
[7] P E Conner, E E Floyd, Differentiable periodic maps, Ergebnisse der Mathematik und ihrer Grenzgebiete, vol. 33, Academic Press Inc. New York; SpringerVerlag, Berlin, (1964)

[8] F T Farrell, L E Jones, Rigidity for aspherical manifolds with $\pi_{1} \subset \mathrm{GL}_{m}(\mathbb{R})$, Asian J. Math. 2 (1998) 215-262

[9] J C Hausmann, On the homotopy of nonnilpotent spaces, Math. Z. 178 (1981) $115-123$

[10] N Higson, G Kasparov, Operator $K$-theory for groups which act properly and isometrically on Hilbert space, Electron. Res. Announc. Amer. Math. Soc. 3 (1997) 131-142

[11] F Hirzebruch, Involutionen auf Mannigfaltigkeiten (German) 1968 Proc. Conf. on Transformation Groups (New Orleans, LA 1967) 148-166

[12] N Keswani, Homotopy invariance of relative eta-invariants and $C^{*}$-algebra $K$-theory, Electron. Res. Announc. Amer. Math. Soc. 4 (1998) 18-26

[13] S Lopez de Medrano, Involutions on manifolds, Ergebnisse der Mathematik und ihrer Grenzgebiete, Band 59, Springer-Verlag, New York-Heidelberg (1971)

[14] W Lück, $L^{2}$-invariants of regular coverings of compact manifolds and $C W-$ complexes, to appear in the Handbook of Geometric Topology

[15] W Lück, $L^{2}$-Invariants: Theory and Applications to Geometry and K-Theory. A Series of Modern Surveys in Mathematics, vol. 44, Springer-Verlag, Berlin (2002)

[16] W Lück, T Schick, Various $L^{2}$-signatures and a topological $L^{2}$-signature theorem, preprint

[17] W Lück, T Schick, Approximating $L^{2}$-signatures by their finite-dimensional analogues, Preprintreihe SFB 478 - Geometrische Strukturen in der Mathematik, Heft 190, Münster (2001)

[18] W Mathai, Spectral flow, eta-invariants and von Neumann algebras, J. Funct. Anal. 109 (1992) 442-456

[19] M Ramachandran, von Neumann index theorems for manifolds with boundary, J. Differential Geom. 38 (1993) 315-349

[20] A Ranicki, Localization in quadratic L-theory, from: "Algebraic topology, Waterloo, 1978 (Proc. Conf., Univ. Waterloo, Waterloo, Ont. 1978)" Lecture Notes in Math. 741, Springer, Berlin(1979) 102-157,

[21] B Vaillant, Indextheorie für Überlangerungen, Diplomarbeit, Universität Bonn, http://styx.math. uni-bonn.de/boris/diplom.html (1997)

[22] C T C Wall, Surgery on compact manifolds, London Mathematical Society Monographs, No. 1, Academic Press, London-New York (1970)

[23] S Weinberger, Homotopy invariance of $\eta$-invariants, Proc. Nat. Acad. Sci. USA 83 (1988) 5362-5363

[24] S Weinberger, The Topological Classification of Stratified Spaces, Chicago Lectures in Mathematics, University of Chicago Press, Chicago, IL (1994) 